$4-2006$

\title{
Feature Selection for Real-Time Tracking
}

D. Frank Hsu

Fordham University

Damian M. Lyons

Fordham University

Jizhou Ai

Fordham University

Follow this and additional works at: https://fordham.bepress.com/frcv_facultypubs

Part of the Robotics Commons

\section{Recommended Citation}

Hsu, D. Frank; Lyons, Damian M.; and Ai, Jizhou, "Feature Selection for Real-Time Tracking" (2006). Faculty Publications. 28. https://fordham.bepress.com/frcv_facultypubs/28

This Article is brought to you for free and open access by the Robotics and Computer Vision Laboratory at DigitalResearch@Fordham. It has been accepted for inclusion in Faculty Publications by an authorized administrator of DigitalResearch@Fordham. For more information, please contact considine@fordham.edu. 


\title{
Feature Selection for Real-Time Tracking
}

\author{
D. Frank Hsu, Damian M. Lyons and Jizhou Ai \\ Robotics \& Computer Vision Laboratory \\ Department of Computer \& Information Science \\ Fordham University, Bronx, NY 10458 \\ \{hsu,lyons,ai\}@cis.fordham.edu
}

\begin{abstract}
We address the problem of selecting features to improve automated video tracking of targets that undergo multiple mutual occlusions. As targets are occluded, different feature subsets and combinations of those features are effective in identifying the target and improving tracking performance. We use Combinatorial Fusion Analysis to develop a metric to dynamically select which subset of features will produce the most accurate tracking. In particular we show that the combination of a pair of features A and B will improve the accuracy only if (a) A and B have relative high performance, and (b) A and B are diverse. We present experimental results to illustrate the performance of the proposed metric.
\end{abstract}

Keywords: video surveillance, target tracking, feature selection, sensory fusion.

\section{INTRODUCTION}

Previous research approaches to recognizing and tracking targets that undergo occlusion have included modeling the target in such a way that occlusion can be recognized and corrected [14] or modeling the statistical nature of the occlusion [28]. However, we note another approach: as a target moves through a series of occlusions in a crowded, urban space, the feature or the set of features that best identifies and tracks the object changes also. In this paper we focus on the issue of dynamically selecting the set of features that improves tracking performance for objects that undergo multiple occlusions.

Feature selection for object recognition has been addressed by several authors ([15], [25]). For tracking, Collin and Liu [4] have looked at the problem of feature selection in terms of finding the single color feature that best distinguishes a target from its background at any point during tracking. Their measure of 'best' is in terms of the largest distance between foreground and background classes as distinguished by that single feature. We will present a method to select the pair of features that produces the best tracking performance at any point during tracking, where by 'best,' we mean that the feature or feature combination yields a target track that is closer to ground truth.

Our principal tool in identifying which features are most useful is the emerging field of Combinatorial Fusion Analysis or CFA ([8]-[13], [19]-[20], [17], [22], [23], [27]). CFA differs from other approaches to data and information fusion [4] in that it considers: (A) both score and rank function for each feature/evidence and explores the interaction between the two functions, (B) both combinatorial possibility and computational efficiency of combining multiple scoring systems, and (C) multiple scoring systems obtained by a variety of different methods such as probability, statistics, analysis, combinatorics and computation. In our work, we (a) adopt CFA to inspect and analyze the space of possible combinations of the features or evidence, (b) explore the scoring behavior of each of the features by computing the rank/score function and propose to use the rank/score function $f_{A}$ to represent the scoring behavior of a feature A, and (d) use the difference between the two rank/score functions $d\left(f_{A}, f_{B}\right)$ to measure the diversity between A and B.

In this paper we present the results of a CFA experiment to select the subset of features that improve tracking performance for a video sequence of two targets undergoing a series of mutual occlusions. We show that the subset of features that improves performance can be predicted at any point during the tracking sequence by considering two criteria: A feature performance ratio $P R(A, B)$, and a feature rank/score diversity $d\left(f_{A}, f_{B}\right)$. 


\section{PRIOR WORK}

There are several key approaches to target recognition and tracking under occlusions. In the contour-based approach ([14], [21]), the issue is how to account for the occluded portion of the contour. In the model-based approach ([18], [28]), since the 'real' appearance of the target (i.e., the model) is known, the issue is to determine how best the occluded view of the target matches its 'real' appearance. In the feature-based approach [16], the crucial problem is determining which features remain robust to occlusion.

Our work falls into the feature-based approach. Lin and Bhanu [16] introduce a feature synthesis strategy for target recognition based on a genetic programming approach. Compositions of primitive features are learned that produce improved discrimination between target classes as long as the targets are not overlapping or occluded. We are specifically interested in overlapping and occluded targets.

Collins and Liu [4] make the point that a tracker is only as good as the features it uses to detect a target. They address the problem that as a target move across a background, the color features (in their case, a subset of linear combinations of the red, green and blue values) that best detect the target change accordingly. On each frame, they search their space of color features to find the one that best distinguished target from background in that frame. Normalized histograms are used to derive discrete probability density functions for each feature on the target and on the background. A variance ratio of the total variance over both foreground and background divided by the sum of the variances within foreground and background is used as a criterion to pick the feature that best distinguishes the foreground from background.

In a series of papers [9],[13], [19],[20] the authors have investigated the problem of tracking on a single camera, using multiple feature cue information, in situations where targets engage in multiple mutual occlusions. The core theory for our approach is based on the work of Hsu, Shapiro and Taksa [11]-[12] on characterizing the scoring behavior, the relationship between the scores assigned by an expert (e.g., a classifier, a filter, etc.) to a list of candidates and the ranks of the candidates. We have developed a framework for fusion for target tracking applications that exploits the combinatorial options for score and rank feature fusion combinations to improve tracking performance. We found that in certain situations, a feature fusion operation that included similarity rank information produced a more accurate track than a score fusion operation using a Mahalanobis sum of similarities [19]. Our conclusion was that the rank information was less sensitive to the effects of target occlusions. However, that research did not provide us with a way to predict at any frame in the video, which fusion of which features would provide the more accurate result. A predictor metric needed to be developed that when applied to the video for a specific target, would indicate which features and which fusion operation would yield the most accurate track.

CFA has been applied to a number of areas, including Information Retrieval (IR), pattern recognition (PR), virtual screening (VS) and drug discovery, and protein structure prediction (PSP) ([7]-[8], [10]-[12], [17], [23], [27]). This previous work has suggested that in a combinatorial setting a combination of multiple features improves on the performance over each of those features only if each of the features itself has good performance and if the features are diverse. In this paper, we quantify performance and diversity, and we present a tracking experiment that explores the performance of all rank and score binary combinations from three features (color, shape and position) to determine the value of the performance ratio and diversity metrics as predictors of the tracking performance a pair of features.

\section{COMBINATORIAL FUSION ANALYSIS}

We consider each feature measured by a sensor (which may measure multiple features) or each piece of the evidence reported by a multiple sensor system as a scoring system for a tracking and recognition module A on the set of $\boldsymbol{n}$ possible tracks or the pool of $\boldsymbol{n}$ track hypotheses, $\boldsymbol{D}=\left\{d_{1}, d_{2}, \ldots, d_{n}\right\}$. Let $s_{A}(x)$ be the scoring function which assigns a real number to each $d_{i}$ in $\boldsymbol{D}$. We view the function $s_{A}(x)$ as the score function with respect to the scoring system (feature/evidence) A from $\boldsymbol{D}$ to $\boldsymbol{R}$ (the set of real numbers). When treating $s_{A}(x)$ as an array of real numbers, it would lead to a rank function $r_{A}(x)$ after sorting the $s_{A}(x)$ array into descending order and assigning a rank (a positive natural number) to each of the $d_{i}$ in $\boldsymbol{D}$. The resulting rank function $r_{A}(x)$ is a function from $\boldsymbol{D}$ to $\boldsymbol{N}=\{1,2, \ldots, n\}$ (we note that $|\boldsymbol{D}|=n)$.

In order to properly compare and correctly combine score functions from multiple scoring systems (multiple features for a single sensor, or multiple items of evidence from multiple sensors) normalization is needed. We simply 
adopt the following transformation from $s_{A}(x): \boldsymbol{D} \rightarrow \boldsymbol{R}$ to $s_{A}(x): \boldsymbol{D} \rightarrow[0,1]$ where $s_{A}(x)=\frac{s_{A}(x)-s_{\min }}{s_{\max }-s_{\min }}, x \in \boldsymbol{D}$ and $s_{\max }=$ $\max \left\{s_{A}(x) \mid x \in \boldsymbol{D}\right\}$ and $s_{\min }=\min \left\{s_{A}(x) \mid x \in \boldsymbol{D}\right\}$.

Given $m$ scoring systems $A_{i}, i=1,2, \ldots, m$, with score functions $s_{A_{i}}(x)$ and rank function $r_{A_{i}}(x)$, there exist several different ways of combining the output of the scoring systems, including score combination, rank combination, voting, average combination and weighted combination. Initially we will define the average rank (or score) combination as follows. For the $m$ scoring systems $A_{i}$ with $s_{A_{i}}(x)$ and $r_{A_{i}}(x)$, we define the score functions $s_{R}$ and $s_{S}$ of the rank combination (RC) and score combination (SC) respectively as:

$$
s_{R}(x)=\sum_{i=1}^{m}\left[r_{A_{i}}(x) / m\right], \quad \text { and } \quad s_{S}(x)=\sum_{i=1}^{m}\left[s_{A_{i}}(x) / m\right] .
$$

As we did before, $s_{R}(x)$ and $s_{S}(x)$ are then sorted into ascending and descending order to obtain the rank function of the rank combination $r_{R}(x)$ and the score combination $r_{R}(x)$, respectively. (In the experiment described later, a Mahalanobis weighted sum is used for score fusion instead of a simple average).

When $m$ scoring systems (features or evidence) $A_{i}, i=1,2, \ldots, m$, together with the score function $s_{A_{i}}(x)$ and rank function $r_{A_{i}}(x)$ are used, combinatorially there are $2^{m}-1\left(=\sum_{k=1}^{m}\left(\begin{array}{l}m \\ k\end{array}\right)\right)$ possible combinations for these $m$ scoring systems using either rank or score functions. The order of complexity is exponential and becomes prohibitive when $m$ is large. The study of multiple scoring systems on large data sets $\boldsymbol{D}$ involves sophisticated mathematical, statistical, and computational approaches and techniques (see e.g., [8] and refs). For example, each of the rank functions of the scoring system $A_{i} i=1,2, \ldots, m$, on $\boldsymbol{D},|\boldsymbol{D}|=n$, can be mapped to a point in the $n$-dimensional polyhedron called the rank space. The $n$-dimensional polyhedron $Q_{n}$ is also a Cayley graph with the symmetric group $S_{n}$ as the vertex set and the adjacency between vertices is defined by a set of generators (a subset of permutations) acting on its vertices.

Remark 1: Previous work in CFA ([7][8], [11]-[12], [17],[23],[27]) in IR, PR, VS and PSP have demonstrated that: (1) the combination of multiple scoring systems (features or evidence) would improve the prediction or classification accuracy rate only if (a) each of the scoring systems has a relatively good performance, and (b) the individual scoring systems are distinctive (or diversified), and (2) rank combinations perform better than score combinations under conditions (a) and (b) and other restrictions.

For the purpose of this paper, our approach considers combinations of two scoring systems selected from the $\left(\begin{array}{l}m \\ 2\end{array}\right)=\frac{m(m-1)}{2}$ possible two combinations using a diversity measure $d(A, B)$ between the scoring systems A and B.

Remark 2: The diversity $d(A, B)$ between $\mathrm{A}$ and $\mathrm{B}$ has been studied using the score functions $d\left(s_{A}, s_{B}\right)$ and rank functions $d\left(r_{A}, r_{B}\right)$ as correlation and rank correlation respectively. The approach of the current proposal is to also use the concept of the rank/score function to measure the diversity between A and B. That is, we include $d\left(f_{A}, f_{B}\right)$ in addition to $d\left(s_{A}, s_{B}\right)$ and $d\left(r_{A}, r_{B}\right)$, where $f_{A}, f_{B}$ are the rank/score functions of $\mathrm{A}$ and $\mathrm{B}$ respectively. The inclusion of $d\left(f_{A}, f_{B}\right)$ in the measurement of the diversity between scoring systems A and B is one of the novelties of our approach.

When plotting the graph of the rank/score function (hence it is called the rank/score graph) of scoring systems $\mathrm{A}$ and $\mathrm{B}$ on the same coordinate plane, the diversity measure can be easily visualized. Different diversity measurements have been considered in other application domains ([2], [6]-[8], [11][13], [17], [23], [27]).

Let $s_{A}(x)$ and $r_{A}(x)$ be the score function and the rank function of the scoring system A. The rank/score function $f_{A}(x): N \rightarrow[0,1]$ is defined as:

$$
f_{A}(i)=\left(s_{A}^{*} \circ r_{A}^{-1}\right)(i)=s_{A}^{*}\left(r_{A}^{-1}(i)\right)
$$

We note that the set $\boldsymbol{N}$ is different from the set $\boldsymbol{D}$ which is the set of $n$ possible tracks or the pool of $n$ track hypotheses. The set $\boldsymbol{N}$ is used as the index set for the rank function value. The rank/score function so defined signifies the scoring (or ranking) behavior of the scoring system and is independent of the tracks or track hypotheses under consideration. Again, the diversity measure $d(A, B)=d\left(f_{A}, f_{B}\right)$ can be defined in several different fashions. Here we use the following: 


$$
d\left(f_{A}, f_{B}\right)=\sum_{i=1}^{n}\left|f_{A}(i)-f_{B}(i)\right|
$$

\section{EXPERIMENTAL INVESTIGATION}

\subsection{Design of Experiment}

The goal of the experiment is to determine whether a combination of a feature diversity measure and a relative performance measure are a good candidate metric for predicting whether a specific pair of features will produce more accurate tracking results or not. The RAF tracking software was constructed (see [9], [13]) to evaluate rank and score fusion operations for multisensory, multitarget video tracking. The three features measured are a color feature, a shape feature and a position feature (see section 4.2 for details). The experiment consisted of running a modified RAF tracking system which calculated and evaluated all pairs of rank and score feature combinations against ground truth data, and comparing the evaluation results with the values of the proposed predictive metric.

There are 11 possible combinations of the three features and two operations: the basic three features (3), the score combination of any two of these (3), the rank combination of any two of these (3) and the rank and score combination of all three features (2). In this paper, six of these, the six pairs, were evaluated, omitting individual features and the combinations of all three features.

Evaluation consisted of comparing at each point the top $q=30$ track hypotheses for each target against the ground truth for the video sequence. Ground truth was obtained by having a human observer mark each target in each video frame (see Figure 1). Each track is compared to the ground truth by evaluating a Mean Sum of Squared Differences (MSSD). The performance measure for a combination A, written $\mathrm{P}(\mathrm{A})$, is inversely proportional to the average MSSD for the top tracks:

$$
P(A)=\frac{q}{1+\sum_{i \leq q} M S S D\left(\text { track }_{i}\right)}
$$

where track $_{i}$ is the ith ranked track hypothesis for combination A.

For each frame, for each target, the six 2-combinations are divided into positive and negative combinations. A combination $\mathrm{A}$ is considered positive if the tracks it produces are closest to the ground truth (i.e., it has the largest $P(A)$ ); all the other pairs are considered negative. The positive combination is the one that should be chosen to improve
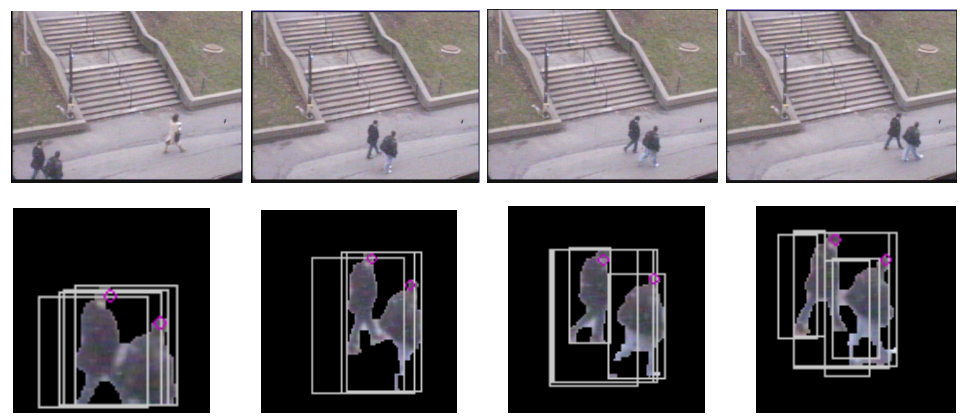

Figure 1: Frames from the video sequence showing two repeatedly occluding targets.

The bottom four images show the foreground regions and bounding boxes extracted for the top four frames. The small circle on each target is the ground truth marker.

tracking performance.

For each combination, two performance metrics are evaluated. The rank-score diversity, calculated for a combination of features $\mathrm{A}$ and $\mathrm{B}$ as

$$
d\left(f_{A}, f_{B}\right)=\sum_{i=1}^{n}\left|f_{A}(i)-f_{B}(i)\right|
$$

and the performance ratio metric, $P R(A, B)$, calculated as: 


$$
P R(A, B)=\frac{\operatorname{MIN}(P(A), P(B))}{\operatorname{MAX}(P(A), P(B))} .
$$

On each step, for each combination, the value of $d\left(f_{A}, f_{B}\right), P R(A, B)$, and whether the combination was positive or negative was recorded to a log file.

The video sequence selected for the experiment shows a group of 2 targets that move at varying speeds in a roughly left to right motion (see Figure 1). The targets undergo repeated mutual occlusions, varying from small to large occlusions. Ground truth for the targets was obtained a frame by frame inspection and annotation by a human observer.

\subsection{RAF Tracker Implementation}

A multisensor, multitarget tracking system, the RAF tracker, was described in [9], [13], [19], and [20]. In that system, foreground regions are extracted from each frame of the image sequence using a non-parametric background estimation technique. The regions are passed to the three component trackers. Color, position and shape information are collected by applying a tracker-specific measurement to each region $c_{j}$ in the frame:

(a). Color Tracker: $m_{c o l}\left(c_{j}\right)$, average normalized RGB color of $c_{j}$.

(b). Location Tracker: $\quad m_{l o c}\left(c_{j}\right)$, image position of the centroid of $c_{j}$.

(c). Shape Tracker: $m_{\text {sha }}\left(c_{j}\right)$, area of the image covered by $c_{j}$ in pixels.

For each frame $i$ in the video sequence, a common MHT based [1][3] hypothesis generation module associates these measurements with the set of existing track hypothesis $\mathbf{D}_{i}$. The gating function is that a track hypothesis be within a standard deviation of the predicted position $p_{k}$ for target $k$ :

$$
\left(p_{k}-m_{\text {loc }}\left(c_{j}\right)\right)^{2}<\sigma_{k}^{2}
$$

Any existing track hypothesis which meets the gating criterion for a region $c_{j}$ is associated with that region, and a new track hypothesis is generated that is the old track extended by this region. Each of the three trackers applies its similarity function to determine how well the region fits that target hypothesis. In addition to the extension of tracks by new measurements, each region also gives rise to a new track of length 1 initialized to a fixed new track score (to model newly appearing targets), and

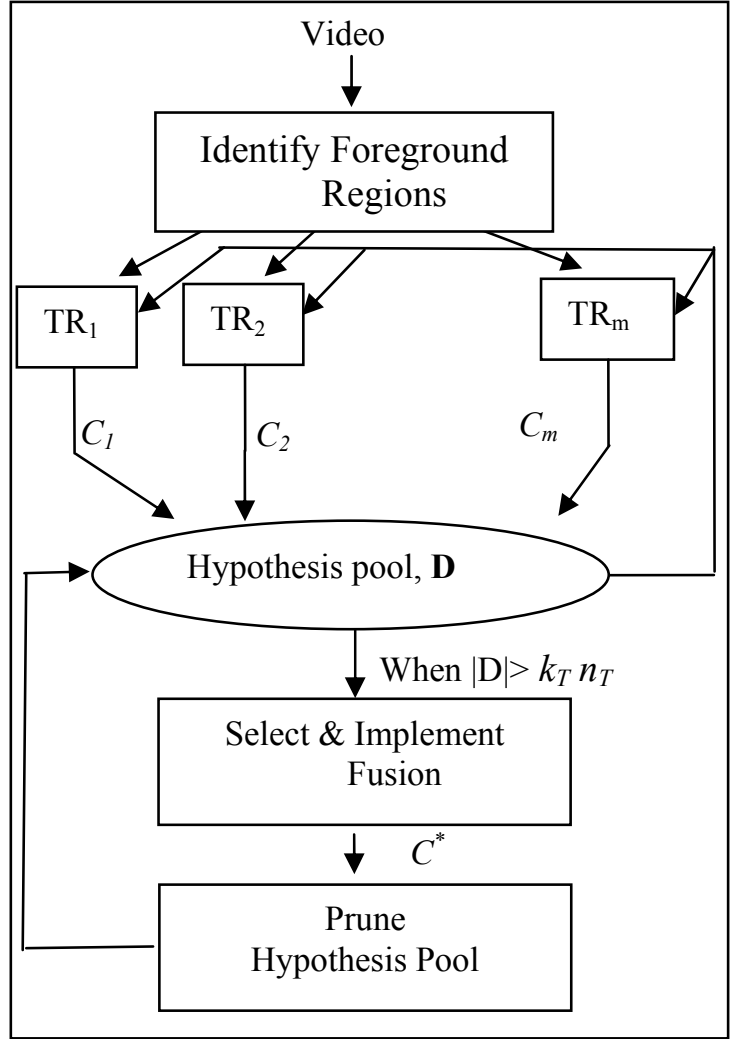

Figure 2: Tracker Block Diagram each track gives rise to a new track of the same length with its score modified by a fixed false alarm score (to model false alarms). The pool of track hypotheses grows as follows:

$$
\left|\boldsymbol{D}_{i+1}\right|=\left|\boldsymbol{D}_{i}\right| \times\left(n_{i}+1\right)+n_{i}
$$

where $n_{i}$ is the number of regions segmented from frame $i$. The set of target to measurement association hypotheses (including new targets and false alarms, and assuming that at most one measurements matches at most one target) is then generated and used to calculate a normalized score value for each track hypothesis.

The pool of track hypotheses grows combinatorially, and needs to be pruned to stay within resource limits. The resource limits are represented by a nominal pool size $n_{T}$ :

$$
\left(\left|\mathbf{D}_{i}\right|>k_{T} n_{T}\right) \Rightarrow \text { Prune } \mathbf{D}_{i} \text { to size } n_{T}
$$

The values $n_{T}=100, k_{T}=2.5$ were used here. The top scoring candidates for all targets after fusion were preserved. To get the best track hypotheses for each target, the scores from each of the separate trackers are fused in all combinations of two from three features using both rank fusion and score fusion. The score fusion operation is a Mahalanobis sum 
(where the coefficients are normalized and inversely proportional to the variance). The rank fusion operation is average rank.

\subsection{Results}

In Figure 3, we show performance graphs for the six feature combinations (3 score and 3 rank) of the 3 features. The performance is the inverse MSSD value, $P(C)$, from section 4.1. These graphs illustrate the fact that at different points during tracking, different feature combinations perform better, i.e., produce tracks that are closer to ground truth.
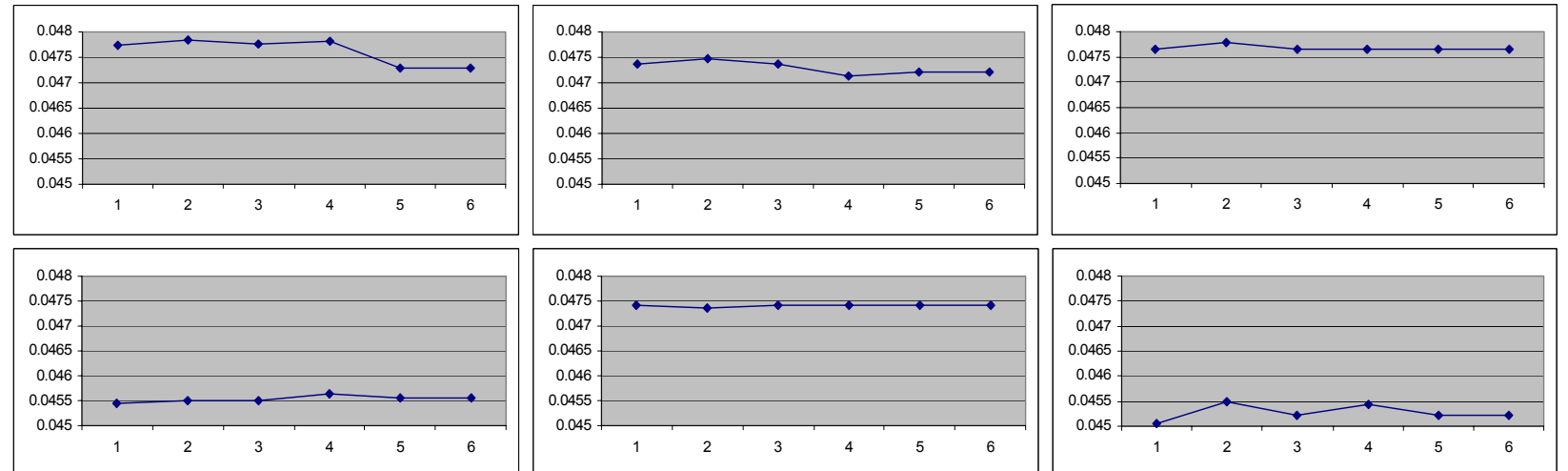

Figure 3: Feature Performance Graphs

These graphs show the six feature pair combinations from six successive evaluations at pruning. The vertical axis is the inverse MSSD for the fused track, and the horizontal axis is the combination (1-3 score, 4-6 rank) of two features from the three color, shape and position features. Combinations $1 \& 4$ are color and position, $2 \& 5$, color and shape, $3 \& 6$, shape and position.

The scatter graph in Figure 4 shows the distribution of positive combinations (best performance) to negative combinations (all others) when plotted in the rank/score diversity and performance ratio space. Looking at the graph, it can be seen that the positive combinations, the combinations producing a result closest to ground truth for each target and for each frame, cluster further up and towards the right, i.e., towards greater diversity and greater performance ratio. The negative combinations, all the other combinations in each case, are widely spread, but cluster strongly in the lower diversity, lower performance ratio part of the space. The centroid of all positive combinations is indicated on the graph with an asterisk marker, and of all negative combinations with an X marker

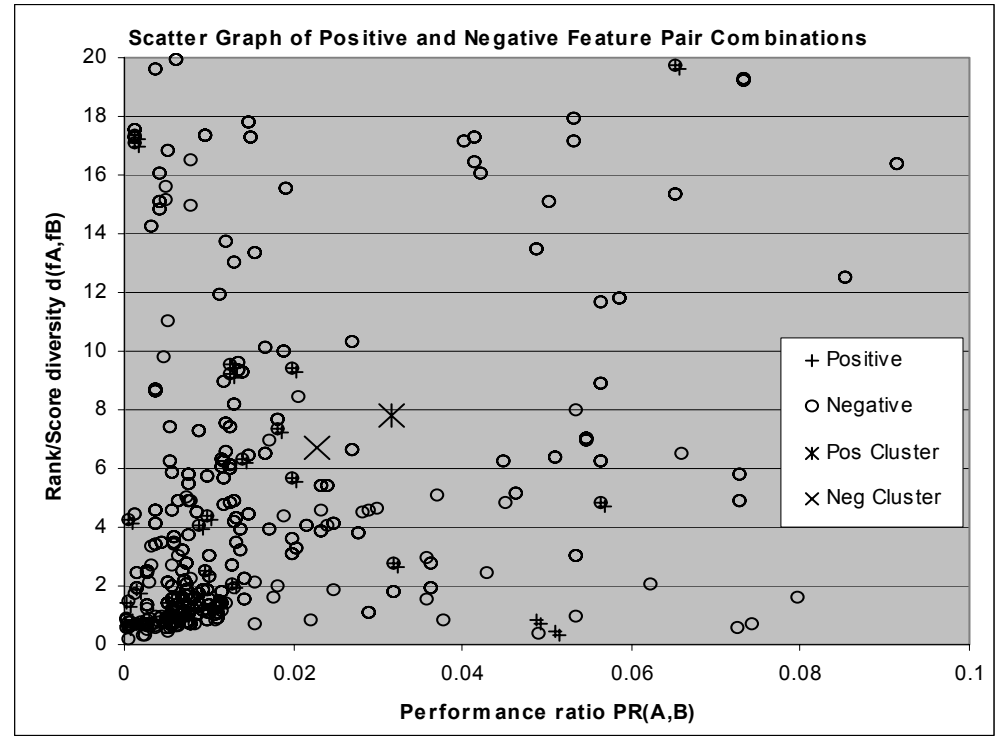

Figure 4: Scatter Graph of Feature Combination Metrics 


\section{CONCLUSION}

In this paper we have addressed the problem of multitarget tracking in which the targets undergo mutual occlusions. Our approach is based on Combinatorial Fusion Analysis, the investigation of the space of all combinations of measured features. As a target moves through occlusions, different combinations of features become necessary to maintain accurate tracking. The specific problem we addressed here is how to select which pair of features produces the best tracking performance. We proposed and evaluated a combined metric, rank/score diversity and relative performance, for predicting the best pair.

We conduct a tracking experiment on a video sequence of two targets moving together with repeated mutual occlusions. We use the RAF tracking system, developed in previous work, as the basis of this experiment. The system is modified to evaluate all combinatorial options for fusing two of the three features of color, shape and position in a rank and a score combination by comparing the quality of the resultant tracks with a ground truth measurement. Combinations are considered positive if the combination performance is closer to ground truth than that of all the other combinations, otherwise the combination is considered negative. The values of the rank/score diversity and performance ratio metrics are measured for each case.

Our results show that the negative combinations tend to cluster when graphed in the area of low rank/score diversity and low relative performance. Thus, more specifically, we show that the combination of a pair of features A and B will improve the accuracy only if (a) A and B have relative high performance, and (b) A and B are diverse. This indicates that the two metrics proposed can be a useful indicator to select those feature combinations 'on the fly' that will improve tracking performance as targets move through multiple occlusions.

\section{REFERENCES}

[1] Bar-Shalom, Y. and Fortmann, T., Tracking and Data Association. 1988: Academic Press.

[2] Brown, G., Wyatt, J., Harris, R., and Yao, X.; Diversity Creation Method: A survey and categorization. Inf. Fusion 6 (2005), pp5-20.

[3] Cox, I.J. and Hingorani, S.L. An Efficient Implementation and Evaluation of Reid's Multiple Hypothesis Tracking Algorithm for Visual Tracking. Int. Conf. on Pattern Recog. (1994) pp.437-442.

[4] Collins, B., and Liu, Y., Online selection of discriminative tracking features. IEEE Int. Conf. on Computer Vision Nice, France, 2003.

[5] Dasarathy, B.V. (Editor); Elucidative Fusion Systems - An Exposition. Information Fusion 1 (2001) pp.5-15.

[6] Kuncheva, L., Diversity in Multiple Classifier Systems. Information Fusion 6(1), March 2005.

[7] Ho, T.K., Hull, J.J., and Srihari, S.N.; Decision Combination in Multiple Classifier Systems, IEEE Trans on Pattern Analysis and Machine Intelligence, 16(1), (1994) pp.66-75.

[8] Hsu, D.F., Chung, Y.S., and Kristel, B.S.; Combinatorial Fusion Analysis: Methods and Practice of Combining Multiple Scoring Systems. In: (H.H. Hsu, editor) Advanced Data Mining Technologies in Bioinformatics, Ideal Group Inc, (2005) in press.

[9] Hsu, D.F., Lyons, D.M., Usandivaras, C., and Montero, F. RAF: A Dynamic and Efficient Approach to Fusion for Multi-target Tracking in CCTV Surveillance. IEEE Int. Conf. on Multisensor Fusion and Integration. Tokyo, Japan; (2003) pp.222-228.

[10]Hsu, D.F., and Palumbo, A., A Study of Data Fusion in Cayley Graphs $\mathrm{G}\left(\mathrm{S}_{\mathrm{n}}, \mathrm{P}_{\mathrm{n}}\right)$, Proc. $7^{\text {th }}$ Int. Symp. On Parallel Architectures, Algorithms and Networks (ISPAN'04), 2004. pp. 557-562.

[11]Hsu, D.F., Shapiro, J., and Taksa, I., Methods of Data Fusion in Information Retreival: Rank vs. Score Combination. 2002, DIMACS TR 2002-58.

[12] Hsu, D.F. and Taksa, I., Comparing rank and score combination methods for data fusion in information retrieval, Information Retrieval 8 (2005). pp.449-480.

[13]Hsu, D.F., and Lyons, D.M., A Dynamic Pruning and Feature Selection Strategy for Real-Time Tracking. 19th IEEE International Conference on Advanced Information Networking and Applications, March 28-30 (2005) pp. 117-124.

[14] Koschan, A., Kang, S., Paik, J., Abidi, B., and Abidi, M., Color active shape models for tracking non-rigid objects. Pattern Recognition Letters 24: pp. 1751-1765, July 2003.

[15] Le, D-D., and Satoh, S., An Efficient Feature Selection Method for Object Detection. Int. Conf. on Advances in Pattern Recognition, Bath UK, 2005.

[16]Lin, Y., Bhanu, B., Object Detection via Feature Synthesis Using MDL-based Genetic Programming. IEEE Trans.

6242-17 V. 2 (p.7 of 8) / Color: No / Format: Letter / Date: 1/22/2006 7:38:56 AM

SPIE USE:

DB Check,

Prod Check, Notes: 
SMC 35(3): pp. 538-547. June 2005.

[17]Lin, C.Y., Lin, K.L., Huang, C.D., Chang, H.M., Yang, C.Y., Lin, C.T., Tang, C.Y., and Hsu, D.F.; Feature Selection and Combination Criteria for improving Predictive Accuracy in Protein Structure Classification. IEEE Symp. On Bioinformatics \& Bioengineering (2005) in press.

[18] Liu, Z., and Sarkar, S., Effect of Silhouette Quality on Hard Problems in Gait Recognition. IEEE Trans. SMC Part B. 35(2): pp.170-182, April 2005.

[19] Lyons, D., and Hsu, D.F., Rank-based Multisensory Fusion in Multitarget Video Tracking. IEEE Intr. Conf. on Advanced Video \& Signal-Based Surveillance. Como, Italy. (2005).

[20] Lyons, D., Hsu, D.F., Usandivaras, C., and Montero, F. Experimental Results from Using a Rank and Fuse Approach for Multi-Target Tracking in CCTV Surveillance. IEEE Intr. Conf. on Advanced Video \& Signal-Based Surveillance. Miami, FL; (2003) pp.345-351.

[21] Mulayim, A.Y., Yilmaz, U., and Ataly, A., Silhouette-based 3D Model Reconstruction from Multiple Images. IEEE Trans. SMC Part B 33(4): pp. 582-591, August 2003.

[22] Melnik, O., Vardi, Y., and Zhang, C-H., Mixed Group Ranks: Preference and Confidence in Classifier Combination. IEEE PAMI V26, N8, August 2004, pp.973-981.

[23] Ng, K.B. and Kantor, P.B.; Predicting the Effectiveness of Naive Data Fusion on the Basis of System Characteristics. J. of Amer. Society for Information Sci. V.51 N.12 (2000), pp1177-1189.

[24] Snidaro, L., Foresti, G., Niu, R., and Varshney, P. Sensor Fusion for Video Surveillance. 7th Int. Conf. on Information Fusion. Stockholm Sweden, (2004) pp.739-746.

[25] Sun, S., Bebis, G., and Miller, R., Object Detection using Feature Subset Selection. Pattern Recognition 37 (2004) pp.2165-2176.

[26] Xu, L., Krzyzak, A., and Suen, C.Y., Method of Combining Multiple Classifiers and their Application to Handwriting Recognition. IEEE Trans. SMC, 22 (3): (1992). pp. 418-435.

[27] Yang, J.M., Chen, Y.F., Shen, T.W., Kristal, B.S., and Hsu, D.F.; Consensus scoring criteria for improving enrichment in virtual screening. J. of Chemical Inf. \& Mod. 45 (2005), pp 1134-1146.

[28] Ying, Z., Castanon, D., Partially occluded object recognition using statistical models. Int. J. of Computer Vision 49(1): pp. 57-78 2002. 